\title{
Suzuki-Type Fixed Point Results in G-Metric Spaces and Applications
}

\author{
Madhu Aggarwal ${ }^{*}$, Renu Chugh and Raj Kamal \\ Department of Mathematics, \\ Maharshi Dayanand University, \\ Rohtak-124001(INDIA)
}

\begin{abstract}
In this paper, we obtain some Suzuki-type fixed point results in G-metric spaces and as well as discuss the G-continuity of the fixed point. The direction of our extension/generalization is new and very simple. An illustrative example is also given to show that our main result is extension of the existing ones. Moreover, we show that these maps satisfy property $\mathrm{P}$. Application to certain class of functional equations arising in dynamical programming is also obtained.
\end{abstract}

\section{Key Words and Phrases}

G-metric space, fixed point, Suzuki contraction, G-continuity, property $\mathrm{P}$, functional equation.

\section{INTRODUCTION}

The classical Banach contraction principle states that "every contraction on a complete metric space admits a unique fixed point". This theorem is very important because it is very forceful tool in nonlinear analysis. Many fixed point theorems have been proved by various authors as generalizations of this result. In 2008, Suzuki [8] introduced a new type of mapping and obtained the following interesting and simple generalization of Banach contraction principle:

Theorem 1.1 [8]: Define a non-increasing function $\theta$ from $[0,1)$ onto $\left(\frac{1}{2}, 1\right]$ by

$\theta(r)= \begin{cases}1 & \text { if } 0 \leq r \leq \frac{1}{2}(\sqrt{5}-1) \\ \frac{1-r}{r^{2}} & \text { if } \frac{1}{2}(\sqrt{5}-1) \leq r \leq \frac{1}{\sqrt{2}} \\ \frac{1}{1+r} & \text { if } \frac{1}{\sqrt{2}} \leq r<1 .\end{cases}$

Let $(X, d)$ be a complete metric space and let $T$ be a mappings from $X$ into itself. Assume that there exists $r \in[0,1)$ such that for every $x, y \in X$

$\theta(r) d(x, T x) \leq d(x, y)$ implies d(Tx, Ty $) \leq \mathrm{rd}(\mathrm{x}, \mathrm{y})$.

Then there exists a unique fixed point $\mathrm{z}$ of $\mathrm{T}$. Moreover, $\lim _{n} T^{n}(\mathrm{x})=\mathrm{z}$ for all $\mathrm{x} \in \mathrm{X}$.

This result attracted several authors to work along these lines and subsequently Theorem 1.1 was generalized and extended in various ways (see for instance [1, 5, and 7]).

In 2006, Z. Mustafa and B. Sims [10] introduced the notion of G-metric spaces as a generalization of the notion of metric spaces in which every triplet of elements is assigned to a nonnegative real number. Mustafa et. al.[12] initiated fixed point theory in this spaces. Later on several authors obtained some fixed point results under various contractive conditions in Gmetric spaces, (see [3, 4, 6, 9, 11-13]).

The aim of this paper is to combine the idea of G-metric space and Suzuki-type fixed point theorems for a single map. Also we prove the uniqueness of the fixed point, as well as the Gcontinuity of the fixed point. The result obtained here extends some existing ones. We also provide an example in support of our main result. Moreover, we show that these maps satisfy property P. An interesting fact about maps satisfying property $\mathrm{P}$ is that none of these maps have any non-trivial periodic points. Some papers dealing with property $\mathrm{P}$ are $[2,6]$. At the end, an application regarding the existence and uniqueness of solutions of certain class of functional equations arising in dynamical programming is obtained.

\section{PRELIMINARIES}

Definition 2.1.[10] Let $\mathrm{X}$ be a nonempty set and let $\mathrm{G}: \mathrm{X} \times \mathrm{X}$ $\times \mathrm{X} \rightarrow \mathrm{R}^{+}$a function satisfying the following axioms:

(G1) $\mathrm{G}(\mathrm{x}, \mathrm{y}, \mathrm{z})=0$ if $\mathrm{x}=\mathrm{y}=\mathrm{z}$,

(G2) $0<\mathrm{G}(\mathrm{x}, \mathrm{x}, \mathrm{y})$ for all $\mathrm{x}, \mathrm{y} \in \mathrm{X}$ with $\mathrm{x} \neq \mathrm{y}$,

(G3) $\mathrm{G}(\mathrm{x}, \mathrm{x}, \mathrm{y}) \leq \mathrm{G}(\mathrm{x}, \mathrm{y}, \mathrm{z})$, for all $\mathrm{x}, \mathrm{y}, \mathrm{z} \in \mathrm{X}$, with $\mathrm{z} \neq \mathrm{y}$,

(G4) $\mathrm{G}(\mathrm{x}, \mathrm{y}, \mathrm{z})=\mathrm{G}(\mathrm{x}, \mathrm{z}, \mathrm{y})=\mathrm{G}(\mathrm{y}, \mathrm{z}, \mathrm{x})=\cdots$ (symmetry in all the three variables),

(G5) $\mathrm{G}(\mathrm{x}, \mathrm{y}, \mathrm{z}) \leq \mathrm{G}(\mathrm{x}, \mathrm{a}, \mathrm{a})+\mathrm{G}(\mathrm{a}, \mathrm{y}, \mathrm{z})$, for all $\mathrm{x}, \mathrm{y}, \mathrm{z}, \mathrm{a} \in \mathrm{X}$, (rectangle inequality).

Then the function $\mathrm{G}$ is called a generalized metric, or, more specifically a $G$-metric on X, and the pair $(\mathrm{X}, \mathrm{G})$ is called a $\mathrm{G}$ metric space.

Definition 2.2[10]. Let $(X, G)$ be a G-metric space, let $\left\{x_{n}\right\}$ be a sequence of points of $\mathrm{X}$, we say that $\left\{\mathrm{x}_{\mathrm{n}}\right\}$ is $G$-convergent to $\mathrm{x}$ if $\lim _{n, m \rightarrow \infty} G\left(x, x_{n}, x_{m}\right)=0$; that is, for any $\varepsilon>0$, there exists $\mathrm{k} \in \mathbf{N}$ such that $\mathrm{G}\left(\mathrm{x}, \mathrm{x}_{\mathrm{n}}, \mathrm{x}_{\mathrm{m}}\right)<\varepsilon$, for all $\mathrm{n}, \mathrm{m} \geq \mathrm{k}$ (throughout this paper we mean by $\mathbf{N}$ the set of all natural numbers). We call $x$ the limit of the sequence and write $x_{n}$ $\rightarrow \mathrm{x}$ or $\lim \mathrm{x}_{\mathrm{n}}=\mathrm{x}$.

Proposition2.3[10].Let $(X, G)$ be a G-metric space. Then the following are equivalent:

(1) $\left\{x_{n}\right\}$ is G-convergent to $x$,

(2) $\mathrm{G}\left(\mathrm{x}_{\mathrm{n}}, \mathrm{x}_{\mathrm{n}}, \mathrm{x}\right) \rightarrow 0$, as $\mathrm{n} \rightarrow \infty$,

(3) $\mathrm{G}\left(\mathrm{x}_{\mathrm{n}}, \mathrm{x}, \mathrm{x}\right) \rightarrow 0$, as $\mathrm{n} \rightarrow \infty$,

(4) $\mathrm{G}\left(\mathrm{x}_{\mathrm{m}}, \mathrm{x}_{\mathrm{n}}, \mathrm{x}\right) \rightarrow 0$, as $\mathrm{m}, \mathrm{n} \rightarrow \infty$.

Definition 2.4[10]. Let $(X, G)$ be a G-metric space. A sequence $\left\{\mathrm{x}_{\mathrm{n}}\right\}$ is called $G$-Cauchy if for each $\varepsilon>0$, there is $\mathrm{k}$ $\in N$ such that $\mathrm{G}\left(\mathrm{x}_{\mathrm{n}}, \mathrm{x}_{\mathrm{m}}, \mathrm{x}_{l}\right)<\varepsilon$, for all $\mathrm{n}, \mathrm{m}, l \geq \mathrm{k}$, that is, if $\mathrm{G}\left(\mathrm{x}_{\mathrm{n}}, \mathrm{x}_{\mathrm{m}}, \mathrm{x}_{l}\right) \rightarrow 0$ as $\mathrm{n}, \mathrm{m}, l \rightarrow \infty$.

Proposition 2.5[10]. Let $(X, G)$ be a G-metric space, then the following conditions are equivalent:

(1) The sequence $\left\{x_{n}\right\}$ is G-Cauchy.

(2) For every $\varepsilon>0$, there exists $\mathrm{k} \in \mathrm{N}$ such that $\mathrm{G}\left(\mathrm{x}_{\mathrm{n}}, \mathrm{x}_{\mathrm{m}}\right.$, $\left.\mathrm{x}_{\mathrm{m}}\right)<\varepsilon$, for all $\mathrm{n}, \mathrm{m} \geq \mathrm{k}$. 
Proposition 2.6[10]. Let $(X, G)$ be a G-metric space. Then $\mathrm{f}$ : $\mathrm{X} \rightarrow \mathrm{X}$ is G-continuous at $\mathrm{x} \in \mathrm{X}$ if and only if it is Gsequentially continuous at $\mathrm{X}$, that is, whenever $\left\{\mathrm{x}_{\mathrm{n}}\right\}$ is $\mathrm{G}$ convergent to $x,\left\{f\left(x_{n}\right)\right\}$ is G-convergent to $f(x)$.

Proposition 2.7[10]. Let $(X, G)$ be a G-metric space. Then the function $\mathrm{G}(\mathrm{x}, \mathrm{y}, \mathrm{z})$ is jointly continuous in all the three of its variables.

Definition 2.8[10].A G-metric space $(\mathrm{X}, \mathrm{G})$ is called $G$ complete if every G-Cauchy sequence is G-convergent in (X, G).

Proposition 2.9[10] Let $(X, G)$ be a G-metric space. Then for any $\mathrm{x}, \mathrm{y}, \mathrm{z}, \mathrm{a} \in \mathrm{X}$ it follows that:

(1) $G(x, y, z)=0$, then $x=y=z$,

(2) $\mathrm{G}(\mathrm{x}, \mathrm{y}, \mathrm{z}) \leq \mathrm{G}(\mathrm{x}, \mathrm{x}, \mathrm{y})+\mathrm{G}(\mathrm{x}, \mathrm{x}, \mathrm{z})$,

(3) $\mathrm{G}(\mathrm{x}, \mathrm{y}, \mathrm{y}) \leq 2 \mathrm{G}(\mathrm{y}, \mathrm{x}, \mathrm{x})$,

(4) $\mathrm{G}(\mathrm{x}, \mathrm{y}, \mathrm{z}) \leq \mathrm{G}(\mathrm{x}, \mathrm{a}, \mathrm{z})+\mathrm{G}(\mathrm{a}, \mathrm{y}, \mathrm{z})$,

(5) $\mathrm{G}(\mathrm{x}, \mathrm{y}, \mathrm{z}) \leq \frac{2}{3}(\mathrm{G}(\mathrm{x}, \mathrm{y}, \mathrm{a})+\mathrm{G}(\mathrm{x}, \mathrm{a}, \mathrm{z})+\mathrm{G}(\mathrm{a}, \mathrm{y}, \mathrm{z}))$,

(6) $\mathrm{G}(\mathrm{x}, \mathrm{y}, \mathrm{z}) \leq(\mathrm{G}(\mathrm{x}, \mathrm{a}, \mathrm{a})+\mathrm{G}(\mathrm{y}, \mathrm{a}, \mathrm{a})+\mathrm{G}(\mathrm{z}, \mathrm{a}, \mathrm{a}))$.

Definition 2.10[10]. A G-metric space $(X, G)$ is called a symmetric G-metric space if

$G(x, y, y)=G(y, x, x)$ for all $x, y \in X$.

Proposition 2.11[10]. Every G-metric space $(X, G)$ defines a metric space space $\left(X, d_{G}\right)$ by

(2.11.1) $\mathrm{d}_{\mathrm{G}}(\mathrm{x}, \mathrm{y})=\mathrm{G}(\mathrm{x}, \mathrm{y}, \mathrm{y})+\mathrm{G}(\mathrm{y}, \mathrm{x}, \mathrm{x})$,

for all $\mathrm{x}, \mathrm{y} \in \mathrm{X}$.

Note that if $(X, G)$ is a symmetric G-metric space, then

(2.11.2) $\quad \mathrm{d}_{\mathrm{G}}(\mathrm{x}, \mathrm{y})=2 \mathrm{G}(\mathrm{x}, \mathrm{y}, \mathrm{y})$, for all $\mathrm{x}, \mathrm{y} \in \mathrm{X}$.

However, if $(X, G)$ is not symmetric, then it holds by the Gmetric properties that

(2.11.3) $\frac{3}{2} \mathrm{G}(\mathrm{x}, \mathrm{y}, \mathrm{y}) \leq \mathrm{d}_{\mathrm{G}}(\mathrm{x}, \mathrm{y}) \leq 3 \mathrm{G}(\mathrm{x}, \mathrm{y}, \mathrm{y})$,

for all $\mathrm{x}, \mathrm{y} \in \mathrm{X}$.

Definition 2.12 [6]. Let $\mathrm{T}$ be a self-mapping of G-metric space with fixed point set $\mathrm{F}(\mathrm{T}) \neq \phi$. Then $\mathrm{T}$ is said to have property $P$ if $\mathrm{F}\left(\mathrm{T}^{\mathrm{n}}\right)=\mathrm{F}(\mathrm{T})$, for each $\mathrm{n} \in \mathrm{N}$. Equivalently, a mapping has property $\mathrm{P}$ if every periodic point is a fixed point.

\section{MAIN RESULTS}

Theorem 3.1. Let $(\mathrm{X}, \mathrm{G})$ be a complete G-metric space and let $\mathrm{T}$ be a mapping on $\mathrm{X}$. Define a strictly decreasing function $\eta$ from $[0,1)$ onto $(1 / 2,1]$ by $\eta(r)=\frac{1}{1+r}$. Assume that there exists $r \in[0,1)$ such that for every $\mathrm{x}, \mathrm{y} \in \mathrm{X}$,

(3.1.1) $\quad \eta(r) \mathrm{G}(\mathrm{x}, \mathrm{Tx}, \mathrm{Tx}) \leq \mathrm{G}(\mathrm{x}, \mathrm{y}, \mathrm{y})$ implies $\mathrm{G}(\mathrm{Tx}, \mathrm{Ty}, \mathrm{Ty}) \leq \mathrm{r} \mathrm{G}(\mathrm{x}, \mathrm{y}, \mathrm{y})$.

Then there exists a unique fixed point $\mathrm{z}$ of $\mathrm{T}$ and $\lim _{n} T^{n}(x)=\mathrm{z}$ for all $\mathrm{x} \in \mathrm{X}$. Moreover, $\mathrm{T}$ is $\mathrm{G}$ continuous at $\mathrm{z}$.

Proof: If $(\mathrm{X}, \mathrm{G})$ is symmetric. From proposition $2.11, \mathrm{~d}_{\mathrm{G}}$ defined by $\mathrm{d}_{\mathrm{G}}(\mathrm{x}, \mathrm{y})=2 \mathrm{G}(\mathrm{x}, \mathrm{y}, \mathrm{y})$ makes $\left(\mathrm{X}, \mathrm{d}_{\mathrm{G}}\right)$ into a metric space. Substituting into (3.1.1) and then multiplying by 2 yield

$\eta(r) \mathrm{d}_{\mathrm{G}}(\mathrm{x}, \mathrm{Tx}) \leq \mathrm{d}_{\mathrm{G}}(\mathrm{x}, \mathrm{y})$ implies $\mathrm{d}_{\mathrm{G}}(\mathrm{Tx}, \mathrm{Ty}) \leq \mathrm{rd}_{\mathrm{G}}(\mathrm{x}, \mathrm{y})$.

From Theorem 1.1, $\mathrm{T}$ has a unique fixed point since

$\eta(r) \leq \theta(r)$ for all $\mathrm{r} \in[0,1)$.

Suppose that $(\mathrm{X}, \mathrm{G})$ is not symmetric. Since $\eta(r) \leq 1$, $\eta(r) \mathrm{G}(\mathrm{x}, \mathrm{Tx}, \mathrm{Tx}) \leq \mathrm{G}(\mathrm{x}, \mathrm{Tx}, \mathrm{Tx})$ holds for every $\mathrm{x} \in \mathrm{X}$. By hypothesis,

(3.1.2) $\quad \mathrm{G}\left(\mathrm{Tx}, \mathrm{T}^{2}(\mathrm{x}), \mathrm{T}^{2}(\mathrm{x})\right) \leq \mathrm{r} \mathrm{G}(\mathrm{x}, \mathrm{Tx}, \mathrm{Tx})$

for all $x \in X$. Let $u \in X$ and define a sequence $\left\{u_{n}\right\}$ in $X$ by $\mathrm{u}_{\mathrm{n}}=\mathrm{T}^{\mathrm{n}} \mathrm{u}$. Then (3.1.2) yields

$$
\mathrm{G}\left(\mathrm{u}_{\mathrm{n}}, \mathrm{u}_{\mathrm{n}+1}, \mathrm{u}_{\mathrm{n}+1}\right)=\mathrm{G}\left(\mathrm{T}^{\mathrm{n}} \mathrm{u}, \mathrm{T}^{\mathrm{n}+1} \mathrm{u}, \mathrm{T}^{\mathrm{n}+1} \mathrm{u}\right) \leq \mathrm{r} \quad \mathrm{G}\left(\mathrm{T}^{\mathrm{n}-1} \mathrm{u}\right.
$$

$\left., \mathrm{T}^{\mathrm{n}} \mathrm{u}, \mathrm{T}^{\mathrm{n}} \mathrm{u}\right) \leq \ldots \ldots \leq \mathrm{r}^{\mathrm{n}} \mathrm{G}(\mathrm{u}, \mathrm{Tu}, \mathrm{Tu})$.

Here $\sum_{n=1}^{\infty} G\left(u_{n}, u_{n+1}, u_{n+1}\right)<\infty$ and $\left\{\mathrm{u}_{\mathrm{n}}\right\}$ is a cauchy

sequence in $\mathrm{X}$. Since $\mathrm{X}$ is complete, so there exists a point $\mathrm{z}$

$\in X$ such that $u_{n} \rightarrow z$. Now, we show that

(3.1.3)

$\mathrm{G}(\mathrm{z}, \mathrm{Tx}, \mathrm{Tx}) \leq \mathrm{r} \mathrm{G}(\mathrm{z}, \mathrm{x}, \mathrm{x})$

for all $\mathrm{x} \in \mathrm{X}$ with $\mathrm{x} \mathrm{z}$

Since $\mathrm{u}_{\mathrm{n}} \rightarrow \mathrm{z}$, there exists $\mathrm{k} \in \mathrm{N}$ with $\mathrm{n} \geq \mathrm{k}$ such that

$\mathrm{G}\left(\mathrm{u}_{\mathrm{n}}, \mathrm{z}, \mathrm{z}\right) \leq \frac{1}{6} \mathrm{G}(\mathrm{x}, \mathrm{z}, \mathrm{z})$ and

$\mathrm{G}\left(\mathrm{u}_{\mathrm{n}}, \mathrm{u}_{\mathrm{n}}, \mathrm{z}\right) \leq \frac{1}{6} \mathrm{G}(\mathrm{x}, \mathrm{z}, \mathrm{z})$ for all $\mathrm{n} \in \mathrm{N}$ with $\mathrm{n} \geq \mathrm{k}$.

Then we have

$$
\begin{aligned}
& \eta(r) \mathrm{G}\left(\mathrm{u}_{\mathrm{n}}, \mathrm{T} \mathrm{u}_{\mathrm{n}}, \mathrm{T} \mathrm{u}_{\mathrm{n}}\right) \leq \mathrm{G}\left(\mathrm{u}_{\mathrm{n}}, \mathrm{T} \mathrm{u}_{\mathrm{n}}, \mathrm{T} \mathrm{u}_{\mathrm{n}}\right) \\
& =\mathrm{G}\left(\mathrm{u}_{\mathrm{n}}, \mathrm{u}_{\mathrm{n}+1}, \mathrm{u}_{\mathrm{n}+1}\right) \\
& \leq \mathrm{G}\left(\mathrm{u}_{\mathrm{n}}, \mathrm{z}, \mathrm{z}\right)+\mathrm{G}\left(\mathrm{z}, \mathrm{u}_{\mathrm{n}+1}, \mathrm{u}_{\mathrm{n}+1}\right) \quad \text { [by (G5)] } \\
& \leq \frac{2}{6} \mathrm{G}(\mathrm{x}, \mathrm{z}, \mathrm{z})=\frac{2}{5}\left[\frac{5}{6} \mathrm{G}(\mathrm{x}, \mathrm{z}, \mathrm{z})\right] \\
& =\frac{2}{5}\left[\mathrm{G}(\mathrm{x}, \mathrm{z}, \mathrm{z})-\frac{1}{6} \mathrm{G}(\mathrm{x}, \mathrm{z}, \mathrm{z})\right] \\
& \leq \frac{2}{5}\left[\mathrm{G}(\mathrm{x}, \mathrm{z}, \mathrm{z})-\mathrm{G}\left(\mathrm{u}_{\mathrm{n}}, \mathrm{z}, \mathrm{z}\right)\right] \\
& \leq \frac{2}{5}\left[G\left(x, u_{n}, u_{n}\right)\right] \leq \frac{4}{5}\left[G\left(u_{n}, x, x\right)\right] \\
& <\mathrm{G}\left(\mathrm{u}_{\mathrm{n}}, \mathrm{x}, \mathrm{x}\right) \text {. }
\end{aligned}
$$

Hence by hypothesis

$\mathrm{G}\left(\mathrm{u}_{\mathrm{n}+1}, \mathrm{Tx}, \mathrm{Tx}\right) \leq \mathrm{rG}\left(\mathrm{u}_{\mathrm{n}}, \mathrm{x}, \mathrm{x}\right)$ for all $\mathrm{n} \geq \mathrm{k}$.

Letting $\mathrm{n} \rightarrow \infty$, we get

$\mathrm{G}(\mathrm{z}, \mathrm{Tx}, \mathrm{Tx}) \leq \mathrm{rG}(\mathrm{z}, \mathrm{x}, \mathrm{x})$ for all $\mathrm{x} \in \mathrm{X}$ with $\mathrm{x} \neq \mathrm{z}$.

Thus (3.1.3) is obtained. We now, prove that $z$ is a fixed point of $\mathrm{T}$. Arguing by contradiction, we assume that $\mathrm{Tz} \neq \mathrm{z}$. We note that for $\mathrm{x}, \mathrm{y} \in \mathrm{X}$, either

(3.1.4) $\quad \eta(r) \mathrm{G}(\mathrm{x}, \mathrm{Tx}, \mathrm{Tx}) \leq \mathrm{G}(\mathrm{x}, \mathrm{y}, \mathrm{y})$

or $\quad \eta(r) \mathrm{G}\left(\mathrm{Tx}, \mathrm{T}^{2}(\mathrm{x}), \mathrm{T}^{2}(\mathrm{x})\right) \leq \mathrm{G}(\mathrm{Tx}, \mathrm{y}, \mathrm{y})$ holds

Now, $\quad \eta(r) \mathrm{G}\left(\mathrm{Tx}, \mathrm{T}^{2}(\mathrm{x}), \mathrm{T}^{2}(\mathrm{x})\right) \leq \mathrm{G}(\mathrm{Tx}, \mathrm{y}, \mathrm{y})$,

implies that, $2 \eta(r) \mathrm{G}\left(\mathrm{Tx}, \mathrm{T}^{2}(\mathrm{x}), \mathrm{T}^{2}(\mathrm{x})\right)$

$$
\leq 2 \mathrm{G}(\mathrm{Tx}, \mathrm{y}, \mathrm{y}) \leq 4 \mathrm{G}(\mathrm{y}, \mathrm{Tx}, \mathrm{Tx}) .
$$

This implies that $\quad \eta(r) \mathrm{G}\left(\mathrm{Tx}, \mathrm{T}^{2}(\mathrm{x}), \mathrm{T}^{2}(\mathrm{x})\right)$

$$
\leq 2 \mathrm{G}(\mathrm{y}, \mathrm{Tx}, \mathrm{Tx}) \text {. }
$$

So, (3.1.4) can be written as

(3.1.5) $\quad \eta(r) \mathrm{G}(\mathrm{x}, \mathrm{Tx}, \mathrm{Tx}) \leq \mathrm{G}(\mathrm{x}, \mathrm{y}, \mathrm{y})$

or $\frac{1}{2} \eta(r) \mathrm{G}\left(\mathrm{Tx}, \mathrm{T}^{2}(\mathrm{x}), \mathrm{T}^{2}(\mathrm{x})\right) \leq \mathrm{G}(\mathrm{y}, \mathrm{Tx}, \mathrm{Tx})$

Indeed if

$$
\eta(r) \mathrm{G}(\mathrm{x}, \mathrm{Tx}, \mathrm{Tx})>\mathrm{G}(\mathrm{x}, \mathrm{y}, \mathrm{y})
$$


and $\frac{1}{2} \eta(r) \mathrm{G}\left(\mathrm{Tx}, \mathrm{T}^{2}(\mathrm{x}), \mathrm{T}^{2}(\mathrm{x})\right)>\mathrm{G}(\mathrm{y}, \mathrm{Tx}, \mathrm{Tx})$,

then we have

$\mathrm{G}(\mathrm{x}, \mathrm{Tx}, \mathrm{Tx}) \leq \mathrm{G}(\mathrm{x}, \mathrm{y}, \mathrm{y})+\mathrm{G}(\mathrm{y}, \mathrm{Tx}, \mathrm{Tx})$

$<\eta(r) \mathrm{G}(\mathrm{x}, \mathrm{Tx}, \mathrm{Tx})+\frac{1}{2} \eta(r) \mathrm{G}\left(\mathrm{Tx}, \mathrm{T}^{2}(\mathrm{x}), \mathrm{T}^{2}(\mathrm{x})\right)$

$\leq \eta(r)\left[\mathrm{G}(\mathrm{x}, \mathrm{Tx}, \mathrm{Tx})+\frac{r}{2} \mathrm{G}(\mathrm{x}, \mathrm{Tx}, \mathrm{Tx})\right]$

$$
=\frac{1+\frac{r}{2}}{1+r} \mathrm{G}(\mathrm{x}, \mathrm{Tx}, \mathrm{Tx})<\mathrm{G}(\mathrm{x}, \mathrm{Tx}, \mathrm{Tx}),
$$

which is a contradiction. Hence either

$\eta(r) \mathrm{G}\left(\mathrm{u}_{2 \mathrm{n}}, \mathrm{u}_{2 \mathrm{n}+1}, \mathrm{u}_{2 \mathrm{n}+1}\right) \leq \mathrm{G}\left(\mathrm{u}_{2 \mathrm{n}}, \mathrm{z}, \mathrm{z}\right)$

or $\eta(r) \mathrm{G}\left(\mathrm{u}_{2 \mathrm{n}+1}, \mathrm{u}_{2 \mathrm{n}+2}, \mathrm{u}_{2 \mathrm{n}+2}\right) \leq \mathrm{G}\left(\mathrm{u}_{2 \mathrm{n}+1}, \mathrm{z}, \mathrm{z}\right)$

holds for every $n \in N$. Using (2.1.1), we obtain that either

$$
\mathrm{G}\left(\mathrm{u}_{2 \mathrm{n}+1}, \mathrm{Tz}, \mathrm{Tz}\right) \leq \mathrm{r} \mathrm{G}\left(\mathrm{u}_{2 \mathrm{n}}, \mathrm{z}, \mathrm{z}\right)
$$

or $\quad \mathrm{G}\left(\mathrm{u}_{2 \mathrm{n}+2}, \mathrm{Tz}, \mathrm{Tz}\right) \leq \mathrm{rG}\left(\mathrm{u}_{2 \mathrm{n}+1}, \mathrm{z}, \mathrm{z}\right)$

holds for every $n \in N$. Since sequence $\left\{u_{n}\right\}$ converges to $z$, the above inequalities imply that there exista a subsequence of $\left\{u_{n}\right\}$ which converges to Tz. This implies that $T z=z$, which is a contradiction. Hence $\mathrm{Tz}=\mathrm{z}$.

We now show that the fixed point is unique. Suppose that $w$ is another fixed point of $\mathrm{T}$.

Since $\quad \eta(r) \mathrm{G}(\mathrm{z}, \mathrm{Tz}, \mathrm{Tz})=0 \leq \mathrm{G}(\mathrm{z}, \mathrm{w}, \mathrm{w})$, we have using (3.1.1) that

$\mathrm{G}(\mathrm{z}, \mathrm{w}, \mathrm{w})=\mathrm{G}(\mathrm{Tz}, \mathrm{Tw}, \mathrm{Tw}) \leq \mathrm{r} \mathrm{G}(\mathrm{z}, \mathrm{w}, \mathrm{w})<\mathrm{G}(\mathrm{z}, \mathrm{w}, \mathrm{w})$,

which is a contradiction. Hence $\mathrm{z}=\mathrm{w}$.

To show that $\mathrm{T}$ is $\mathrm{G}$-continuous at $\mathrm{z}$, let $\left\{\mathrm{y}_{\mathrm{n}}\right\}$ be any sequence in $X$ such that $\left\{y_{n}\right\}$ is G-convergent to $z$. For $n \in N$, we have

$$
\eta(r) \mathrm{G}(\mathrm{z}, \mathrm{Tz}, \mathrm{Tz})=0 \leq \mathrm{G}\left(\mathrm{z}, \mathrm{y}_{\mathrm{n}}, \mathrm{y}_{\mathrm{n}}\right) .
$$

Thus using (2.1.1), we get

$\mathrm{G}\left(\mathrm{z}, T \mathrm{~T}_{\mathrm{n}}, T \mathrm{~T}_{\mathrm{n}}\right)=\mathrm{G}\left(\mathrm{Tz}, T \mathrm{Ty}_{\mathrm{n}}, T \mathrm{~T}_{\mathrm{n}}\right) \leq \mathrm{r} \mathrm{G}\left(\mathrm{z}, \mathrm{y}_{\mathrm{n}}, \mathrm{y}_{\mathrm{n}}\right)$.

Letting $\mathrm{n} \rightarrow \infty$, we get

$$
\lim _{n \rightarrow \infty} G\left(z, T y_{n}, T y_{n}\right)=0
$$

Hence $\left\{\mathrm{Ty}_{\mathrm{n}}\right\}$ is G-convergent to $\mathrm{z}=\mathrm{Tz}$. So $\mathrm{T}$ is G-continuous at $\mathrm{z}$.

The following result is Theorem 5.1.7 of [11] and Corollary 3.4 of [9].

Corollary 3.2. Let $(\mathrm{X}, \mathrm{G})$ be a complete G-metric space and let $\mathrm{T}$ be a mapping on $\mathrm{X}$. Assume there exists $r \in[0,1)$ such that for every $\mathrm{x}, \mathrm{y} \in \mathrm{X}$,

(3.2.1) $\quad \mathrm{G}(\mathrm{Tx}, \mathrm{Ty}, \mathrm{Ty}) \leq \mathrm{r} \mathrm{G}(\mathrm{x}, \mathrm{y}, \mathrm{y})$.

Then there exists a unique fixed point $\mathrm{z}$ of $\mathrm{T}$ and $\lim _{n} T^{n}(x)=\mathrm{z}$ for all $\mathrm{x} \in \mathrm{X}$. Moreover, $\mathrm{T}$ is G-continuous at $\mathrm{z}$.

Proof: It follows directly from Theorem 3.1.1.

Corollary 3.3. Let $(\mathrm{X}, \mathrm{G})$ be a complete G-metric space and let $\mathrm{T}$ be a mapping on $\mathrm{X}$. Define a strictly decreasing function $\eta$ from $[0,1)$ onto $(1 / 2,1]$ by $\eta(r)=\frac{1}{1+r}$. Assume that there exists $r \in[0,1)$ such that for every $\mathrm{x}, \mathrm{y} \in \mathrm{X}$,

$$
\begin{aligned}
& \eta(r) \mathrm{G}\left(\mathrm{x}, \mathrm{T}^{\mathrm{m}} \mathrm{x}, \mathrm{T}^{\mathrm{m}} \mathrm{x}\right) \leq \mathrm{G}(\mathrm{x}, \mathrm{y}, \mathrm{y}) \text { implies } \\
& \mathrm{G}\left(\mathrm{T}^{\mathrm{m}} \mathrm{x}, \mathrm{T}^{\mathrm{m}} \mathrm{y}, \mathrm{T}^{\mathrm{m}} \mathrm{y}\right) \leq \mathrm{r} \mathrm{G}(\mathrm{x}, \mathrm{y}, \mathrm{y}) .
\end{aligned}
$$

Then there exists a unique fixed point $\mathrm{z}$ of $\mathrm{T}$. Moreover, $\mathrm{T}^{\mathrm{m}}$ is G-continuous at $\mathrm{z}$.
Proof. From Theorem 3.1, we conclude that the maps $T^{\mathrm{m}}$ has a unique fixed point say z. Now, $T z=T\left(T^{m} z\right)=T^{m+1} z=$ $\mathrm{T}^{\mathrm{m}}(\mathrm{Tz})$, meaning that $\mathrm{Tz}$ is also a fixed point of $\mathrm{T}^{\mathrm{m}} \cdot$ By the uniqueness of $\mathrm{z}$, we get $\mathrm{Tz}=\mathrm{z}$.

The following example illustrates our main result.

Example 3.4 Let $X=\{(0,0),(0,4),(4,0),(0,5),(5,0),(4$,

$5),(5,4)\}$.

Define G: X

$\times \mathrm{X} \times \mathrm{X} \rightarrow \mathrm{R}^{+}$by

$\mathrm{G}\left[\left(\mathrm{x}_{1}, \mathrm{x}_{2}\right),\left(\mathrm{y}_{1}, \mathrm{y}_{2}\right),\left(\mathrm{z}_{1}, \mathrm{z}_{2}\right)\right]=\max \left\{\mathrm{d}\left[\left(\mathrm{x}_{1}, \mathrm{x}_{2}\right),\left(\mathrm{y}_{1}, \mathrm{y}_{2}\right)\right], \mathrm{d}\left[\left(\mathrm{y}_{1}\right.\right.\right.$, $\left.\left.\left.\mathrm{y}_{2}\right),\left(\mathrm{z}_{1}, \mathrm{z}_{2}\right)\right], \mathrm{d}\left[\left(\mathrm{x}_{1}, \mathrm{x}_{2}\right),\left(\mathrm{z}_{1}, \mathrm{z}_{2}\right)\right]\right\}$,

where $\mathrm{d}: \mathrm{X} \times \mathrm{X} \rightarrow \mathrm{R}^{+}$is defined as

$\mathrm{d}\left[\left(\mathrm{x}_{1}, \mathrm{x}_{2}\right),\left(\mathrm{y}_{1}, \mathrm{y}_{2}\right)\right]=\left|x_{1}-y_{1}\right|+\left|x_{2}-y_{2}\right|$.

Let $\mathrm{T}$ be such that

$\mathrm{T}\left(\mathrm{x}_{1}, \mathrm{x}_{2}\right)=\left\{\begin{array}{c|lc}\left(x_{1}, 0\right) & \text { if } & x_{1} \leq x_{2} \\ \left(0, x_{2}\right) & \text { if } & x_{1}>x_{2}\end{array}\right.$

Then $\mathrm{T}$ does not satisfy the condition (3.2.1) of Corollary 3.2 at $\mathrm{x}=(4,5), \mathrm{y}=(5,4)$. However, all the hypotheses of Theorem 3.1 are satisfied for the map $\mathrm{T}$ and $(0,0)$ is unique fixed point of $\mathrm{T}$.

Remark.3.5 The example 3.4 does not satisfy the conditions of Corollary 3.2 so from it we cannot say that $\mathrm{T}$ has a fixed point or not, but from Theorem 3.1 we can say that $\mathrm{T}$ has a unique fixed point in X. Therefore Theorem 3.1 is a proper generalization of Theorem 5.1.7 of [11] and Corollary 3.4 of [9].

\section{PROPERTY P.}

In this section, we shall show that maps satisfying (3.1.1) possess property $\mathrm{P}$.

Theorem 4.1. Under the conditions of Theorem 3.1, $\mathrm{T}$ has property $P$.

Proof: From Theorem 3.1, T has a fixed point. Therefore, F $\left(\mathrm{T}^{\mathrm{n}}\right) \neq \phi$, for each positive integer $\mathrm{n} \geq 1$. Fix a positive integer $\mathrm{n}>1$ and let $\mathrm{z} \in \mathrm{F}\left(\mathrm{T}^{\mathrm{n}}\right)$. We claim that $\mathrm{z} \in \mathrm{F}(\mathrm{T})$, that is, $\mathrm{z}$ is a fixed point of $\mathrm{T}$. Suppose that $\mathrm{z} \neq \mathrm{Tz}$. Then

$\eta(r) \mathrm{G}\left(\mathrm{T}^{\mathrm{n}-1} \mathrm{z}, \mathrm{T}^{\mathrm{n}} \mathrm{z}, \mathrm{T}^{\mathrm{n}} \mathrm{z}\right) \leq \mathrm{G}\left(\mathrm{T}^{\mathrm{n}-1} \mathrm{z}, \mathrm{T}^{\mathrm{n}} \mathrm{z}, \mathrm{T}^{\mathrm{n}} \mathrm{z}\right)$.

Using (3.1.1), we have

$\mathrm{G}\left(\mathrm{T}\left(\mathrm{T}^{\mathrm{n}-1} \mathrm{z}\right), \mathrm{T}\left(\mathrm{T}^{\mathrm{n}} \mathrm{z}\right), \mathrm{T}\left(\mathrm{T}^{\mathrm{n}} \mathrm{z}\right)\right) \leq \mathrm{r} \mathrm{G}\left(\mathrm{T}^{\mathrm{n}-1} \mathrm{z}, \mathrm{T}^{\mathrm{n}} \mathrm{z}, \mathrm{T}^{\mathrm{n}} \mathrm{z}\right)$

that is, $G\left(T^{n} z, T^{n+1} z, T^{n+1} z\right) \leq r G\left(T^{n-1} z, T^{n} z, T^{n} z\right)$.

Continuing like this, we have

$\mathrm{G}\left(\mathrm{T}^{\mathrm{n}} \mathrm{z}, \mathrm{T}^{\mathrm{n}+1} \mathrm{z}, \mathrm{T}^{\mathrm{n}+1} \mathrm{z}\right) \leq r^{n} \mathrm{G}(\mathrm{z}, \mathrm{Tz}, \mathrm{Tz})$,

That is, $\mathrm{G}(\mathrm{z}, \mathrm{Tz}, \mathrm{Tz}) \leq r^{n} \mathrm{G}(\mathrm{z}, \mathrm{Tz}, \mathrm{Tz})$, which is possible only if $\mathrm{G}(\mathrm{z}, \mathrm{Tz}, \mathrm{Tz})=0$,

that is, $\quad z=T z$. Thus $z \in F(T)$. Hence $T$ satisfies property $P$.

\section{APPLICATION TO FUNCTIONAL EQUATIONS}

In this section we prove existence and uniqueness of solution for a class of functional equations in G-metric space using theorem 3.1.

Let $\mathrm{U}$ and $\mathrm{V}$ be Banach spaces, $\mathrm{W} \subset \mathrm{U}, \mathrm{D} \subset \mathrm{V}$ and $\mathrm{R}$ is the field of real numbers. Let $X=B(W)$ denote the set of all bounded real valued functions on W. Define G: $\mathrm{X} \times \mathrm{X} \times \mathrm{X} \rightarrow$ $\mathrm{R}^{+}$by

$\mathrm{G}\left(\mathrm{h}_{1}, \mathrm{~h}_{2}, \mathrm{~h}_{3}\right)=\max \left\{\mathrm{d}\left(\mathrm{h}_{1}, \mathrm{~h}_{2}\right), \mathrm{d}\left(\mathrm{h}_{2}, \mathrm{~h}_{3}\right), \mathrm{d}\left(\mathrm{h}_{3}, \mathrm{~h}_{1}\right)\right\}$

where $\mathrm{d}: \mathrm{X} \times \mathrm{X} \rightarrow \mathrm{R}^{+}$is defined as 
$\mathrm{d}\left(\mathrm{h}_{1}, \mathrm{~h}_{2}\right)=\sup _{t \in W}\left\{\left|h_{1}(t)-h_{2}(t)\right|\right\}$

then $(\mathrm{X}, \mathrm{G})$ is $\mathrm{G}$-complete metric space. Consider the following functional equation

$\mathrm{p}(\mathrm{x})=\sup _{y \in D}\{g(x, y)+M(x, y, p(\tau(x, y)))\}$,

$\mathrm{x} \in \mathrm{W}$,

where $\mathrm{g}: \mathrm{W} \times D \rightarrow R$ and $\mathrm{M}: \mathrm{W} \times D \times \mathrm{R} \rightarrow R$ are bounded functions. We consider $\mathrm{W}$ and $\mathrm{D}$ as the state and decision spaces, respectively, $\quad \tau: W \times D \rightarrow W \quad$ represents transformation of the process and $\mathrm{p}(\mathrm{x})$ represents the optimal return function with initial state $\mathrm{x}$.

Let a function $\eta$ be defined as in Theorem 3.1 and the mapping $\mathrm{T}: \mathrm{X} \rightarrow \mathrm{X}$ by

$\mathrm{T}(\mathrm{h}(\mathrm{x}))=\sup _{y \in D}\{g(x, y)+M(x, y, h(\tau(x, y)))\}$,

$\mathrm{x} \in \mathrm{W}, \mathrm{h} \in \mathrm{X}$.

Now we prove the existence and uniqueness of the solution of the functional equation (5.1.1).

Theorem 5.1 Suppose that there exists a $\mathrm{r} \in[0,1)$ such that for every

$(\mathrm{x}, \mathrm{y}) \in \mathrm{W} \times D, \mathrm{~h}_{1}, \mathrm{~h}_{2} \in \mathrm{X}, \mathrm{t} \in \mathrm{W}$, the inequality

(5.1.3) $\quad \eta(r) \mathrm{G}\left(\mathrm{h}_{1}, \mathrm{Th}_{1}, \mathrm{Th}_{1}\right) \leq \mathrm{G}\left(\mathrm{h}_{1}, \mathrm{~h}_{2}, \mathrm{~h}_{2}\right) \quad$,

implies that

$$
\left|M\left(x, y, h_{1}(t)\right)-M\left(x, y, h_{2}(t)\right)\right| \leq r\left|h_{1}(t)-h_{2}(t)\right|
$$

holds, then the functional equation (5.1.1) has a unique bounded solution in $\mathrm{X}$.

Proof Let $\lambda$ be an arbitrary positive real number and $h_{1}$, $\mathrm{h}_{2} \in X$. For $\mathrm{x} \in \mathrm{W}$, we choose $\mathrm{y}_{1}, \mathrm{y}_{2} \in \mathrm{D}$ so that

(5.1.5) $\mathrm{T}\left(\mathrm{h}_{2}(\mathrm{x})\right)<\mathrm{g}\left(\mathrm{x}, \mathrm{y}_{2}\right)+\mathrm{M}\left(\mathrm{x}, \mathrm{y}_{2}, \mathrm{~h}_{2}\left(\tau_{2}\right)\right)+\lambda$,

where $\tau_{1}=\tau\left(\mathrm{x}, \mathrm{y}_{1}\right)$ and $\tau_{2}=\tau\left(\mathrm{x}, \mathrm{y}_{2}\right)$.

From the definition of mapping $\mathrm{T}$, we have

(5.1.6) $\quad \mathrm{T}\left(\mathrm{h}_{1}(\mathrm{x})\right) \geq \mathrm{g}\left(\mathrm{x}, \mathrm{y}_{2}\right)+\mathrm{M}\left(\mathrm{x}, \mathrm{y}_{2}, \mathrm{~h}_{1}\left(\tau_{2}\right)\right)$,

(5.1.7) $\quad \mathrm{T}\left(\mathrm{h}_{2}(\mathrm{x})\right) \geq \mathrm{g}\left(\mathrm{x}, \mathrm{y}_{1}\right)+\mathrm{M}\left(\mathrm{x}, \mathrm{y}_{1}, \mathrm{~h}_{2}\left(\tau_{1}\right)\right)$,

If the inquality (5.1.3) holds, then from (5.1.4) and (5.1.7), we obtain

$$
\begin{aligned}
& T\left(h_{1}(x)\right)-T\left(h_{2}(x)\right) \\
& <M\left(x, y_{1}, h_{1}\left(\tau_{1}\right)\right)-M\left(x, y_{1}, h_{2}\left(\tau_{1}\right)\right)+\lambda \\
& \leq\left|M\left(x, y, h_{1}\left(\tau_{1}\right)\right)-M\left(x, y, h_{2}\left(\tau_{1}\right)\right)\right|+\lambda \\
& \leq r\left|h_{1}(x)-h_{2}(x)\right|+\lambda
\end{aligned}
$$

Similarly, from (5.1.2), (5.1.5) and (5.1.6), we obtain

$$
T\left(h_{2}(x)\right)-T\left(h_{1}(x)\right) \leq r\left|h_{1}(x)-h_{2}(x)\right|+\lambda
$$

Hence from (5.1.8) and (5.1.9) we have

$$
\left|T\left(h_{1}(x)\right)-T\left(h_{2}(x)\right)\right| \leq r\left|h_{1}(x)-h_{2}(x)\right|+\lambda
$$

Since the inequality (5.1.10) is true for all $\mathrm{x} \in \mathrm{W}$ and arbitrary $\lambda>0$, then we have

$\eta(r) \mathrm{G}\left(\mathrm{h}_{1}, \mathrm{Th}_{1}, \mathrm{Th}_{1}\right) \leq \mathrm{G}\left(\mathrm{h}_{1}, \mathrm{~h}_{2}, \mathrm{~h}_{2}\right) \quad$ implies that $\mathrm{G}\left(\mathrm{Th}_{1}\right.$, $\left.\mathrm{Th}_{1}, \mathrm{Th}_{2}\right) \leq r \mathrm{G}\left(\mathrm{h}_{1}, \mathrm{~h}_{1}, \mathrm{~h}_{2}\right)$.

Thus all the conditions of Theorem 3.1 are satisfied for the mapping $\mathrm{T}$ and hence the functional equation (5.1.1) has a unique bounded solution.

\section{CONCLUSION}

From the above discussion, it is clear that our results are quite simple, general and includes several fixed point theorems in metric spaces and G-metric spaces as special cases. Further the results of this paper may be extended to a pair of maps, three and more maps in G-metric spaces.

\section{REFERENCES}

[1] G. Mot, and A. Petrusel, "Fixed point theory for a new type of contractive multivalued operators," Nonlinear Analysis: Theory, Methods \& Appl., vol. 70, no. 9, pp. 3371-3377, 2009.

[2] G. S. Jeong and B. E. Rhoades, Maps for which $\mathrm{F}(\mathrm{T})=$ $\mathrm{F}\left(\mathrm{T}^{\mathrm{n}}\right)$, Fixed point theory and applications, vol. 6, (2004), 71-105

[3] M. Abbas and B. E. Rhoades, Common fixed point results for noncommuting mappings without continuity in generalized metric spaces, Applied Math. and Comp.,215 (2009), 262-269.

[4] M. Gugnani, M. Aggarwal and R. Chugh, Common Fixed Point Results in G-Metric Spaces and Applications, Int. Journal of Computer Appl., Vol. 43, No. 11, April 2012, 38-42.

[5] O. Popescu, "Two fixed point theorems for generalized contractionswith constants in complete metric, space," Central European Journal of Mathematics, vol. 7, no. 3, pp. 529-538, 2009.

[6] R. Chugh, T. Kadian, A. Rani and B. E. Rhoades, Property P in G-metric spaces, Fixed Point Theory and App., Volume 2010, Article ID 401684, (2010), 12 pages.

[7] S. Dhompongsa and H. Yingtaweesittikul, "Fixed points for multivalued mappings and the metric completeness," Fixed Point Theory and applications, vol. 2009, Article ID 972395, 15 pages, 2009.

[8] T. Suzuki, A generalized banach contraction principle that characterizes metric completeness, Proceedings of the American mathematical Society, Vol. 136, No. 5, May 2008, 1861-1869.

[9] W. Shatanawi, Fixed point theory for contractive Mappings satisfying $\Phi$-maps in G-metric spaces, Fixed Point Theory and Applications, Volume 2010 ,Article ID 181650,(2010), 9pages.

[10] Z. Mustafa and B. Sims, A new approach to generalized metric spaces, Journal of Nonlinear and Convex Analysis, vol. 7, no. 2, (2006), 289-297.

[11] Z. Mustafa, A new structure for generalized metric space with applications to fixed point theory, $\mathrm{Ph}$. D. Thesis, University of Newcastle, Newcastle, UK, 2005.

[12] Z. Mustafa, H. Obiedat and F. Awawdeh, Some fixed point theorem for mapping on complete G-metric spaces, Fixed Point Theory and Appl., Vol. 2008, Article ID 189870,(2008), 12pages.

[13] Z. Mustafa, W. Shatanawi, M. Bataineh, Existence of fixed point results in G-metric spaces, International Journal of Mathematics and

Mathematical Sciences, vol. 2009, Article ID 283028, 10 pages, 2009. 